\title{
Dimensionamento amostral para analisar caracteres físicos e químicos de frutos de maracujá-fedorento
}

\author{
Edilson Romais Schmildt ${ }^{*}$, Rodrigo Sobreira Alexandre ${ }^{2}$, André Lucas Siqueira ${ }^{1}$, \\ Lívia Giro Mayrinck ${ }^{1}$, Omar Schmildt ${ }^{1}$
}

10.1590/0034-737X201764020002

\section{RESUMO}

Objetivou-se, com este trabalho, determinar o tamanho de amostra para análise de caracteres físicos e químicos de frutos maduros de maracujá-fedorento (Passiflora foetida). Foram colhidos, aleatoriamente, 150 frutos de plantas cultivadas em casa de vegetação. Em laboratório, esses frutos foram mensurados quanto a 12 caracteres (comprimento longitudinal; diâmetro equatorial; massa de fruto; massa de casca; massa de polpa; rendimento de polpa; peso de sementes por fruto; número de sementes por fruto; massa de sementes por fruto; acidez titulável; sólidos solúveis e ratio), sendo calculadas as medidas de tendência central e de dispersão e verificada sua normalidade. Determinou-se o intervalo de confiança bootstrap percentil, a partir da simulação de tamanho 4.000, para 150 tamanhos de amostra (1, $2, \ldots, 150$ frutos) de cada caractere mensurado. Os caracteres físicos e químicos analisados em frutos maduros de $P$. foetida apresentam diferentes precisões experimentais e requerem diferentes tamanhos de amostra. Para o comprimento longitudinal, por exemplo, o diâmetro equatorial e a relação entre estes, seis frutos são suficientes para a estimação da média com um erro de estimação de $5 \%$ da média. Se forem considerados todos os caracteres físicos e químicos de frutos maduros, analisados neste trabalho, são necessários no mínimo 39 frutos, assumindo-se erro de 10\% da estimativa da média.

Palavras-chave: Passiflora foetida var. glaziovii Killip; planejamento experimental; amostragem.

\section{ABSTRACT}

\section{Sample dimension for evaluating physical and chemical characters of wild passion fruit}

The objective of this study was to determine the sample size required to evaluate physical and chemical characters of Passiflora foetida fruits. A hundred and fifty fruits were randomly harvested from plants grown in a greenhouse. In the laboratory, these fruits were measured for 12 characters (length, equatorial diameter, fruit mass, bark mass, pulp mass, pulp yield, seed weight per fruit, number of seeds per fruit, seed mass per fruit, titratable acidity, soluble solids, and ratio) and the measures of central tendency and dispersion were calculated and checked for normality. The bootstrap percentile confidence interval was determined, from the simulation of 4,000, for 150 sample sizes $(1,2, \ldots, 150$ fruits) measured for each character. The physical and chemical characteristics evaluated in mature fruits of $P$. foetida exhibit different experimental accuracies and require different sample sizes. To measure only the longitudinal length, equatorial diameter and the relationship among these six fruits are sufficient to estimate the average estimation error with a 5\% average. If we consider all the physical and chemical characters of ripe fruit in this work, at least 39 fruits are required, assuming $10 \%$ error of the estimated average.

Key words: Passiflora foetida var. glaziovii Killip; experimental planning; sampling.

\footnotetext{
Recebido em 07/11/2014 e aprovado em 23/02/2017.

${ }^{1}$ Universidade Federal do Espírito Santo, Departamento de Ciências Agrárias e Biológicas, São Mateus, Espírito Santo, Brasil. e.romais.s@gmail.com; andresiqueira15@hotmail.com; lgmayrinck@hotmail.com; omar-schmildt@ig.com.br

${ }^{2}$ Universidade Federal do Espírito Santo, Departamento de Ciências Florestais e da Madeira, Jerônimo Monteiro, Espírito Santo, Brasil. rodrigosobreiraalexandre@gmail.com "Autor para correspondência: e.romais.s@gmail.com
} 


\section{INTRODUÇÃO}

O maracujazeiro-fedorento (Passiflora foetida L.) (Marostega et al., 2013) é uma espécie autocompatível (García \& Hoc, 1998), uma das 142 espécies de Passifloraceae identificadas no Brasil (Bernacci et al., 2015). Recentemente, $P$. foetida var. glaziovii foi detectada em área de preservação da estação experimental da Universidade Federal do Espírito Santo, em São Mateus, sendo os acessos avaliados quanto à germinação e ao vigor (Costa, 2013).

Diferentes órgãos de plantas de $P$. foetida L., como folhas, ramos, raízes, frutos e sementes, têm uso terapêutico na medicina popular, utilizados no combate a asma, insônia, emenagogo, biliousnes, histeria, inflamação de pele, ansiedade nervosa, dor de cabeça e erisipelas (Ambasta, 1986; Mohanasundari et al., 2007; Rasool et al., 2011; Bhosale \& Sutar, 2015). O embasamento científico desses usos vem sendo estabelecido ao longo dos anos. Um caso é o da eficiência do uso antibacteriano de extratos de folhas e frutos (Mohanasundari et al., 2007; Bhosale \& Sutar, 2015), sendo que os principais metabólitos secundários encontrados em folhas, ramos, frutos e sementes são alcaloides, flavonoides, compostos glicosídeos cardíacos, antraquinonas e saponinas (Bhosale \& Sutar, 2015). Tricomas presentes principalmente nas flores, frutos, brácteas e estípulas, secretam resina que apresenta defesa contra lagartas de Dione juno juno (Boiça Júnior et al., 2008).

Apesar da vasta utilidade de $P$. foetida, a caracterização física e química de frutos dessa espécie foi encontrada apenas nos trabalhos de Aular \& Rodríguez (2003) e de Soares et al. (2011), tendo sido usados 400 e 15 frutos por acesso avaliado, respectivamente. Portanto, ainda não houve uma padronização quanto ao critério tamanho da amostra, para caracterização de frutos de $P$. foetida.

Em pesquisas agrícolas, é importante o dimensionamento da amostra necessária para a estimação da média de determinada variável, quando a população não pode ser mensurada ou o trabalho demanda tempo, recursos financeiros e humanos excessivos (Toebe et al., 2011). O tamanho de amostra é diretamente proporcional à variabilidade dos dados e à confiabilidade desejada na estimativa, sendo inversamente proporcional ao erro de estimação permitido, a priori, pelo pesquisador (Zar, 2010; Bussab \& Morettin, 2012). Quanto maior for o tamanho de amostra, maior será a precisão do experimento, com redução da variância da média amostral, embora a demanda por recursos também seja elevada. Por outro lado, o tamanho pequeno da amostra pode reduzir a precisão experimental (Zar, 2010).

O tamanho de amostra para avaliação de caracteres de frutos tem sido determinado para várias espécies como o melão (Nunes et al., 2006), laranja (Avanza et al., 2010), pêssego (Toebe et al., 2011; 2012), maçã (Toebe et al., 2011), pimenteira (Silva et al., 2011), maracujá amarelo (Coelho et al., 2011) e maracujá do mato (P. caerulea) (Bandeira et al., 2016). Contudo, trabalhos que determinam o tamanho de amostra necessário para avaliação de caracteres físico-químicos de frutos maduros de $P$. foetida não foram encontrados na literatura, apesar da existência de trabalhos sobre a quantificação de seus caracteres físico-químicos (Aular \& Rodríguez, 2003; Soares et al., 2011). O interesse pela espécie $P$. foetida pode aumentar, em função da descoberta de seus elevados potenciais agronômicos, como a resistência ao Fusarium solani e $F$. oxysporum f. sp. (Preisigke et al., 2015), o que pode recomendar seu uso em cruzamentos com espécies comerciais em programas de melhoramento, além do consumo in natura de seus frutos (Aular \& Rodríguez, 2003).

Assim, objetivou-se, com este trabalho, determinar o tamanho de amostra necessário para a análise de caracteres físico-químicos de frutos maduros de $P$. foetida var. glaziovii.

\section{MATERIAL E MÉTODOS}

O trabalho de dimensionamento amostral foi feito a partir de 15 acessos de P. foetida var. glaziovii Killip, cujo banco de sementes é mantido no laboratório de melhoramento de plantas da Universidade Federal do Espírito Santo (UFES), após confirmação da ocorrência desta espécie no município de São Mateus-ES (39 51'22''S e $\left.18^{\circ} 40^{\prime} 16^{\prime \prime} \mathrm{W}\right)$, por Costa (2013). Foram avaliados $150 \mathrm{fru}-$ tos de 15 plantas cultivadas em casa de vegetação, dez frutos de cada acesso. O critério de maturação de frutos foi adotado conforme Aular \& Rodríguez (2003). Em julho de 2013, seis meses após o plantio, colheram-se aleatoriamente de frutos maduros de cada planta os quais foram avaliados quanto a caracteres físicos $(\mathrm{CL}=$ comprimento longitudinal, em mm; DE = diâmetro equatorial, em mm; $\mathrm{MF}=$ massa de fruto, em g; $\mathrm{MC}=$ massa de casca, em g; $\mathrm{MP}=$ massa de polpa, em g; REND = rendimento de polpa, em $\%$; PS = peso de sementes por fruto, em g; NS = número de sementes por fruto; $\mathrm{MS}=$ massa de sementes por fruto, em g) e químicos (AT = acidez titulável, medido em $\%$ de ácido cítrico; $\mathrm{SS}=$ sólidos solúveis, em ${ }^{\circ}$ Brix; e ratio $=\mathrm{SS} / \mathrm{AT}$ ).

A partir dos dados de cada caractere físico e químico dos 150 frutos de P. foetida var. glaziovii, foram calculadas as estatísticas: valores mínimo e máximo, média aritmética, desvio padrão, coeficiente de variação, assimetria e curtose. Também foi verificada a normalidade dos dados, por meio do teste de Lilliefors. Essas estatísticas foram calculadas com a finalidade de caracterizar o banco de dados e verificar a sua adequação para o estudo do 
dimensionamento amostral, por método determinístico, ou a necessidade de uso de método por simulação bootstrap.

Para as simulações, em cada caractere, utilizou-se a estimação intervalar via bootstrap pelo intervalo percentil (Martinez \& Louzada Neto, 2001; Ferreira, 2009). Foram planejados 150 tamanhos de amostra para cada caractere, sendo o tamanho de amostra inicial de um fruto e, os demais, obtidos com incrementos de um até atingir 150 frutos.

Para cada tamanho de amostra planejado de cada caractere, foram realizadas 4.000 simulações, por meio de reamostragem, com reposição (Martinez \& Louzada Neto, 2001). Para cada amostra simulada, foi estimada a média. Assim, para cada tamanho de amostra de cada caractere, foram obtidas 4.000 estimativas da média (Ferreira, 2009). Depois, calculou-se a amplitude do intervalo de confiança de $95 \%$ (IC 95\%) pela diferença entre o percentil 97,5\% e o $2,5 \%$ para cada tamanho de amostra, sendo esses resultados plotados graficamente. A seguir, determinou-se o tamanho de amostra (número de frutos) para a estimação da média de cada caractere. Para essa determinação, partiu-se do tamanho inicial (um fruto) e considerou-se como tamanho de amostra o número de frutos a partir do qual as médias mantiveram-se dentro do limite do intervalo de confiança a 95\% (Toebe et al., 2011).

As análises estatísticas foram realizadas com o programa R (R Development Core Team, 2010) e, os gráficos, com o aplicativo Microsoft Office Excel ${ }^{\circledR}$ (Levine et al., 2012).

\section{RESULTADOS E DISCUSSÃO}

As medidas de tendência central, de variabilidade, de assimetria e curtose e o teste de Lilliefors, em relação aos caracteres físico-químicos, revelaram que metade dos caracteres com dados dos 150 frutos de P. foetida var. glaziovii aderiram à distribuição normal (Tabela 1). Os caracteres físicos, como massa de fruto (MF), massa de casca (MC), rendimento (REND) e massa de sementes por fruto (MS) não apresentaram distribuição normal, apresentando assimetria positiva da distribuição dos dados (Tabela 1). Os caracteres químicos, como sólidos solúveis (SS) e ratio também apresentaram desvio da normalidade. Diante dessas considerações, como nem todos os caracteres apresentam distribuição normal, determinouse o tamanho da amostra a partir de método por simulação bootstrap percentil, considerando-se que o uso desse procedimento não necessita de pressupostos sobre a distribuição de probabilidade do estimador (Martinez \& Louzada Neto, 2001).

Os valores médios de cada caractere estão próximos dos observados por Aular \& Rodríguez (2003) e por Soares et al. (2011) para diferentes acessos de P. foetida.

Neste trabalho, os caracteres de fácil mensuração, como comprimento longitudinal (CL), diâmetro equatorial (DE) e relação CL/DE, apresentaram coeficientes de variação (CV) inferiores a 10\% (Tabela 1), o que os classifica como de alta precisão (Storck et al., 2011). O maior valor de CV (31,78\%) foi obtido para o caractere ratio, considerado alto e de baixa precisão experimental. Os demais

Tabela 1: Média, mínimo, máximo, desvio padrão (DP), coeficiente de variação (CV\%), assimetria (AS), curtose + 3 (CT) e resultados do teste de normalidade (Lilliefors, L), para 12 caracteres mensurados em 150 frutos de $P$. foetida var. glaziovii

\begin{tabular}{|c|c|c|c|c|c|c|c|c|}
\hline Caractere $^{(1)}$ & Média & Mínimo & Máximo & DP & CV\% & $\mathbf{A} \mathbf{S}^{(2)}$ & $\mathrm{CT}^{(3)}$ & $\mathbf{L}^{(4)}$ \\
\hline \multicolumn{9}{|l|}{ Físico } \\
\hline $\mathrm{CL}$ & 19,464 & 16,390 & 22,440 & 1,101 & 5,65 & $0,089^{\text {ns }}$ & $3,262^{\mathrm{ns}}$ & $\mathrm{S}$ \\
\hline $\mathrm{DE}$ & 17,018 & 13,450 & 19,630 & 1,081 & 6,35 & $-0,150^{\mathrm{ns}}$ & $3,181^{\mathrm{ns}}$ & S \\
\hline $\mathrm{CL} / \mathrm{DE}$ & 1,146 & 0,999 & 1,337 & 0,067 & 5,85 & $0,277^{\mathrm{ns}}$ & $3,067^{\mathrm{ns}}$ & $\mathrm{S}$ \\
\hline $\mathrm{MF}$ & 1,892 & 1,509 & 2,632 & 0,258 & 13,63 & $0,621^{*}$ & $2,711^{\mathrm{ns}}$ & $\mathrm{N}$ \\
\hline $\mathrm{MC}$ & 0,608 & 0,396 & 0,915 & 0,120 & 19,69 & $0,731^{*}$ & $2,829^{\mathrm{ns}}$ & $\mathrm{N}$ \\
\hline MP & 1,046 & 0,497 & 1,501 & 0,195 & 18,68 & $-0,198^{\mathrm{ns}}$ & $3,539^{\mathrm{ns}}$ & $\mathrm{S}$ \\
\hline REND & 53,162 & 34,463 & 88,758 & 10,735 & 20,19 & $0,933^{*}$ & $3,684^{\mathrm{ns}}$ & $\mathrm{N}$ \\
\hline MS & 0,236 & 0,175 & 0,350 & 0,029 & 12,23 & $0,396^{*}$ & $3,825^{*}$ & $\mathrm{~S}$ \\
\hline NS & 19.44 & 7,000 & 29,000 & 4,969 & 25.56 & $-0,413^{*}$ & $2,639^{\mathrm{ns}}$ & $\mathrm{S}$ \\
\hline \multicolumn{9}{|l|}{ Químico } \\
\hline $\mathrm{AT}$ & 1,197 & 0,484 & 2,963 & 0,305 & 25,48 & $1,313^{*}$ & $9,596^{*}$ & $\mathrm{~S}$ \\
\hline SS & 12,777 & 7,000 & 16,000 & 1,656 & 12,96 & $-0,873^{*}$ & $3,678^{\mathrm{ns}}$ & $\mathrm{N}$ \\
\hline ratio & 11,439 & 4,388 & 26,865 & 3,635 & 31,78 & $1,045^{*}$ & $4,791^{*}$ & $\mathrm{~N}$ \\
\hline
\end{tabular}

${ }^{(1)} \mathrm{CL}=$ comprimento longitudinal, em $\mathrm{mm}$; DE = diâmetro equatorial, em mm; MF = massa de fruto, em g; MC = massa de casca, em g; MP = massa de polpa, em g; REND = rendimento de polpa, em \%; MS = massa de sementes por fruto, em g; NS = número de sementes por fruto; $\mathrm{AT}=$ acidez titulável, medido em $\%$ de ácido cítrico; $\mathrm{SS}=$ sólidos solúveis, em ${ }^{\circ}$ Brix; ratio $=\mathrm{SS} / \mathrm{AT}$.

(2)* Assimetria difere de zero pelo teste t, a $5 \%$ de probabilidade. ns = não-significativo.

(3)* Curtose difere de três pelo teste $\mathrm{t}$, a $5 \%$ de probabilidade. $\mathrm{ns}=$ não-significativo.

(4) $\mathrm{S}=$ Distribuição normal, pelo teste de Lilliefors $(\mathrm{P}>0,05) . \mathrm{N}=$ Não normal $(\mathrm{P} \leq 0,05)$. 
caracteres apresentaram CV entre $13,63 \%$ para massa de fruto (MF) e 25,48\% para acidez titulável (AT). Isso sugere que para a obtenção da estimativa da média, com uma determinada precisão, o caractere ratio apresentará o maior tamanho de amostra, e os caracteres CL, DE e relação CL/ DE apresentarão os menores tamanhos de amostra. Segundo Soares et al. (2011), na caracterização de frutos de $P$. foetida, o CV variou entre 4,49\%, para $\mathrm{pH}$, e $42,84 \%$, para massa das sementes, sendo que, para ratio, o $\mathrm{CV}$ foi de $40,16 \%$.
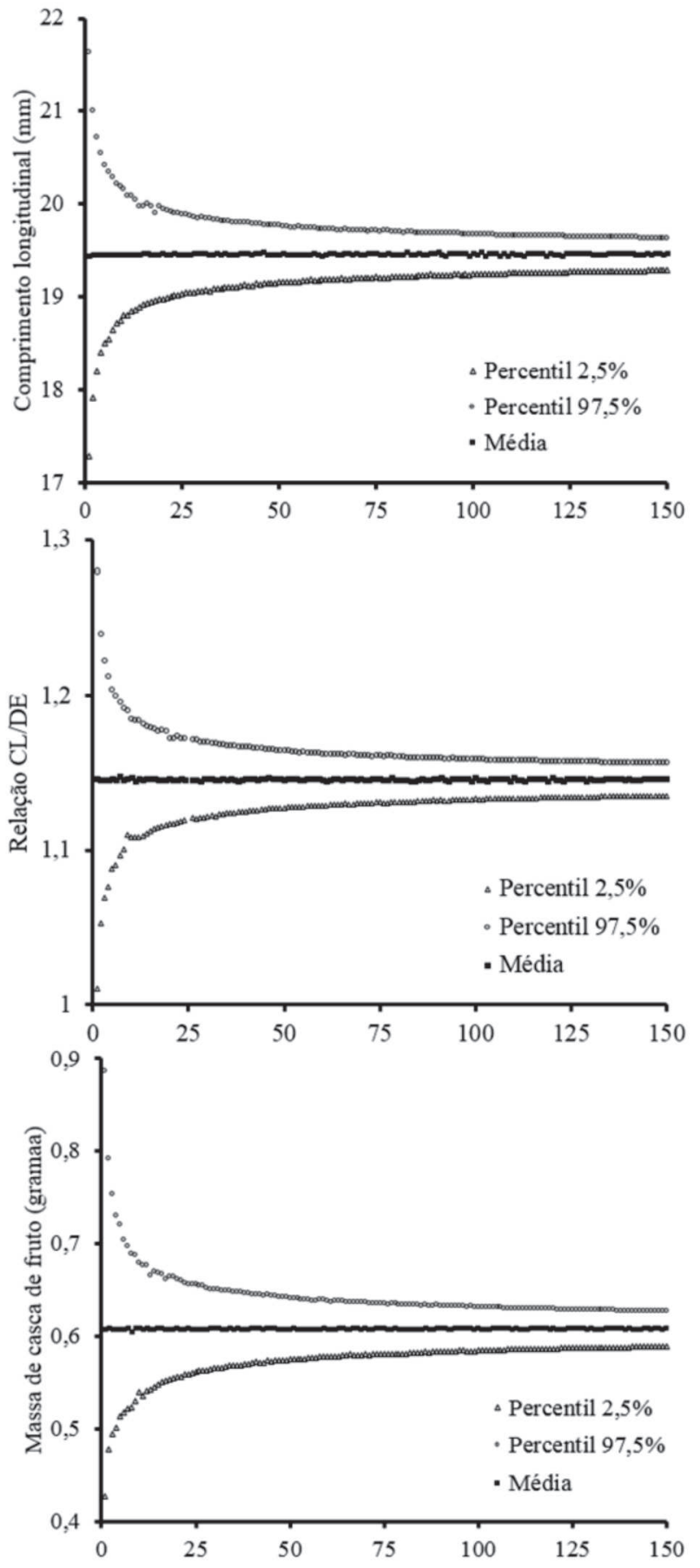

Número de frutos
A amplitude do intervalo de confiança de $95 \%$ diminuiu gradativamente com o acréscimo do tamanho da amostra (número de frutos), o que revela aumento da precisão na estimação da média de cada caractere dos frutos (Figura 1). Nota-se também que a média bootstrap estimada para cada tamanho de amostra é invariável (Martinez \& Louzada Neto, 2001), o que credencia a análise gráfica para determinação do tamanho da amostra de cada caractere, para diferentes erros amostrais assumidos em torno da média.
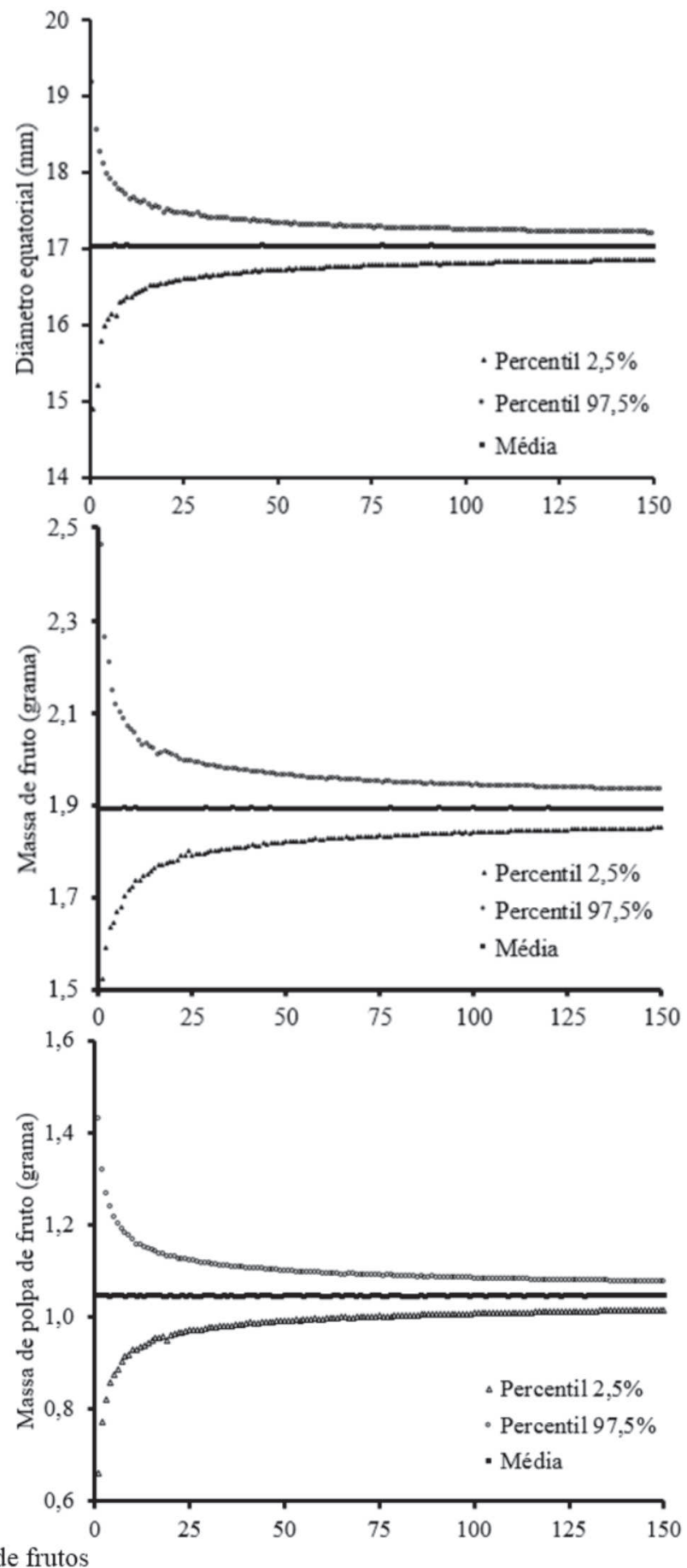

Continua na próxima página... 
Yontinuação da Figura 1
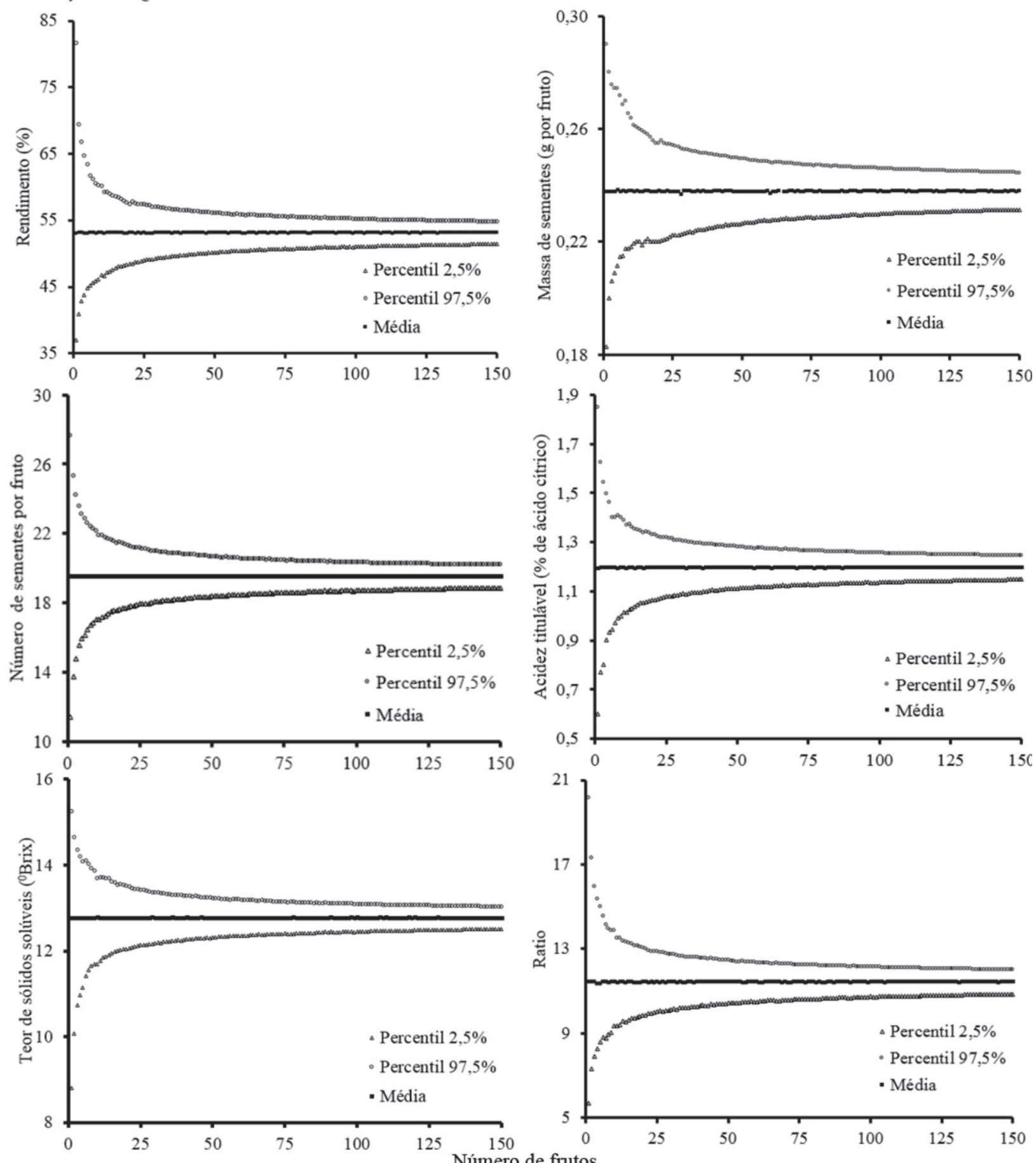

Figura 1: Percentil 2,5\%, percentil 97,5\% e média das 4.000 médias de caracteres físicos e químicos de frutos maduros, obtidos por reamostragens em 150 diferentes tamanhos de amostra $(1,2,3, \ldots 150$ frutos $)$ de $P$. foetida var. glaziovii.

Na Tabela 2, são apresentados os dimensionamentos amostrais de cada caractere de acordo com o erro assumido. Nota-se que um mínimo de cinco frutos é suficiente para estimar CL, DE e relação CL/DE, com erro de estimação igual a 5\% da média. Para este mesmo erro de estimação, são necessários mais de 150 frutos para estimar ratio. Aumentando o erro para 10 e $20 \%$, são necessários no mínimo 39 e dez frutos respectivamente.
Neste trabalho, sete frutos são requeridos para determinar MF com $10 \%$ em torno da média. Trabalhando com maracujá amarelo (P. edulis), Coelho et al. (2011) determinaram que para MF são necessários 16 frutos, com erro de $10 \%$ em torno da média. Também com erro de 10\% em torno da média, Bandeira et al. (2016) trabalhando com $P$. caerulea, determinaram que para MF são necessários 55 frutos. Este menor tamanho da amostra, 
Tabela 2: Número de frutos para a estimação da média de 12 características de frutos maduros de P. foetida var. glaziovii, para amplitudes do intervalo de confiança de $95 \%$

\begin{tabular}{|c|c|c|c|c|c|c|c|c|}
\hline \multirow{2}{*}{ Caractere $^{(1)}$} & \multicolumn{8}{|c|}{ Amplitudes do intervalo de confiança de $95 \%$} \\
\hline & $5 \%$ & $6 \%$ & $7 \%$ & $8 \%$ & $9 \%$ & $10 \%$ & $15 \%$ & $20 \%$ \\
\hline \multicolumn{9}{|l|}{ Físico } \\
\hline$\overline{C L}$ & 5 & 3 & 3 & 2 & 2 & 1 & 1 & 1 \\
\hline DE & 6 & 4 & 3 & 2 & 2 & 2 & 1 & 1 \\
\hline $\mathrm{CL} / \mathrm{DE}$ & 5 & 4 & 3 & 2 & 2 & 1 & 1 & 1 \\
\hline MF & 29 & 20 & 15 & 11 & 9 & 7 & 3 & 2 \\
\hline $\mathrm{MC}$ & 61 & 42 & 31 & 24 & 19 & 15 & 7 & 4 \\
\hline MP & 54 & 38 & 28 & 21 & 17 & 14 & 6 & 3 \\
\hline REND & 64 & 44 & 32 & 24 & 19 & 16 & 7 & 4 \\
\hline PS & 46 & 32 & 23 & 18 & 14 & 11 & 5 & 3 \\
\hline NS & 68 & 47 & 35 & 27 & 21 & 12 & 5 & 3 \\
\hline \multicolumn{9}{|l|}{ Químico } \\
\hline $\mathrm{AT}$ & 100 & 69 & 51 & 39 & 31 & 25 & 11 & 6 \\
\hline SS & 26 & 18 & 13 & 10 & 8 & 6 & 3 & 2 \\
\hline ratio & $>150$ & 108 & 79 & 61 & 48 & 39 & 17 & 10 \\
\hline
\end{tabular}

(1) $\mathrm{CL}=$ comprimento longitudinal, em $\mathrm{mm} ; \mathrm{DE}=$ diâmetro equatorial, em $\mathrm{mm}$; $\mathrm{MF}=$ massa de fruto, em $\mathrm{g}$; $\mathrm{MC}=\mathrm{massa}$ de casca, em $\mathrm{g}$; $\mathrm{MP}=$ massa de polpa, em g; REND = rendimento de polpa, em \%; PS = peso de sementes por fruto, em g; NS = número de sementes por fruto; MS = massa de sementes por fruto, em g; $\mathrm{AT}=$ acidez titulável, medido em $\%$ de ácido cítrico; $\mathrm{SS}=$ sólidos solúveis, em ${ }^{\circ}$ Brix; ratio $=$ SS/AT.

verificado para $P$. foetida poderia ser atribuído à condição de cultivo, visto que, neste trabalho, as plantas foram cultivadas em casa de vegetação e, nos trabalhos de Coelho et al. (2011) e de Bandeira et al. (2016), os frutos usados para avaliação foram obtidos de plantas a campo. No entanto, por se tratar de espécies diferentes, devese considerar que não apenas as condições ambientais, mas também e principalmente, que o genótipo influencia na variabilidade (Aular \& Rodríguez, 2003). Reforça-se o fato de P. foetida ser autocompatível, diferente de P. edulis (García \& Hoc, 1998) e, que, neste trabalho, as plantas dos 15 acessos produziram frutos com sementes no ambiente casa de vegetação.

\section{CONCLUSÕES}

A avaliação dos caracteres físicos e químicos de frutos maduros de $P$. foetida requer diferentes graus de precisão experimental e diferentes tamanhos de amostra.

Para a mensuração de apenas caracteres físicos, seis frutos são suficientes para a estimação da média, com um erro de estimação de $5 \%$ da média.

Se forem considerados conjuntamente caracteres físicos e químicos de frutos maduros avaliados neste trabalho, são necessários no mínimo 39 frutos, assumindo-se erro de $10 \%$ da estimativa da média.

\section{AGRADECIMENTOS}

Os autores agradecem à CAPES e ao CNPq, pelo apoio na forma de bolsas de estudo.

\section{REFERÊNCIAS}

Ambasta SP (1986) The useful plants of India. New Delhi, Publication and Information Directorate, CSIR. 918p.

Aular J \& Rodríguez Y (2003) Algunas características físicas y químicas del fruto de cuatro especies de Passiflora. Bioagro, 15:41-46.

Avanza MM, Bramardi SJ \& Mazza SM (2010) Tamaño óptimo de muestra para evaluar el patrón de crecimiento de frutos de naranjo 'Valencia Iate'. Revista Brasileira de Fruticultura, 32:1154-1163.

Bandeira CT, Fortes SKG, Toebe M, Salfert L, Giacobbo GL \& Welter LJ (2016) Sample size for estimate the average of Passiflora caerulea fruits traits. Ciência Rural, 46:1729-1736.

Bernacci LC, Cervi AC, Milward-de-Azevedo MA, Nunes TS, Imig DC, Mezzonato AC (2015) Passifloraceae in Lista de Espécies da Flora do Brasil. Jardim Botânico do Rio de Janeiro. Disponível em: <http://www.floradobrasil.jbrj.gov.br/jabot/floradobrasil/ FB12506>. Acesso em 27 Mar 2017.

Boiça Júnior AL, Angelini MR \& Oliveira JC (2008) Aspectos biológicos de Dione juno juno (Cramer) (Lepidoptera: Nymphalidae) em genótipos de maracujazeiro. Revista Brasileira de Fruticultura, 30:101-105.

Bhosale U \& Sutar V (2015) Quantitative, qualitative, phytochemical estimation and antioxidant activities of different parts of Passiflora foetida. Indian Streams Research Journal, 5:1-7.

Bussab WO \& Morettin PA (2012) Estatística básica. $7^{\mathrm{a}}$ ed. São Paulo, Saraiva. 540p.

Coelho AA, Oliveira EMS, Resende ED \& Thiébaut JTL (2011) Dimensionamento amostral para caracterização da qualidade pós-colheita do maracujá amarelo. Revista Ceres, 58:23-28.

Costa PR (2013) Morfometria, germinação in vitro e ex vitro e adequação metodológica do teste de tetrazólio em sementes de Passiflora foetida var. glaziovii killip (Passifloraceae). Dissertação de Mestrado. Universidade Federal do Espírito Santo, São Mateus. 56p. 
Ferreira DF (2009) Estatística básica. $2^{\mathrm{a}}$ ed. Lavras, UFLA. 664p.

García MTA \& Hoc PS (1998) Biologia floral de Passiflora foetida (Passifloraceae). Revista de Biologia Tropical, 46:191-202.

Levine DM, Stephan DF, Krehbiel TC \& Berenson ML (2012) Estatística: Teoria e aplicações usando Microsoft Excel em português. $6^{a}$ ed. Rio de Janeiro, LTC Editora. 832p.

Marostega TN, Ferraz ACL, Araújo LM, Luz PB, Sobrinho SP \& Neves LG (2013) Superação de dormência em sementes de Passiflora foetida L. Perspectiva, 37:57-64.

Martines EZ \& Louzada Neto F (2001) Estimação intervalar via bootstrap. Revista de Matemática e Estatística, 19:217-251.

Mohanasundari C, Natarajan D, Srinivasan K, Umamaheswari S \& Ramachandran A (2007) Antibacterial properties of Passiflora foetida L.: a common exotic medicinal plant. African Journal of Biotechnology, 6:2650-2653.

Nunes GHS, Torquato JE, Sales Júnior R, Ferreira HA \& Bezerra Neto F (2006) Tamanho amostral para estimar o teor de sólidos solúveis totais em talhões de melão amarelo. Revista Caatinga, 19:117-122.

Preisigke SC, Martini FV, Rossi AAB, Serafim ME, Barelli MAA, Luz PB, Araújo KL \& Neves LG (2015) Genetic variability of Passiflora spp. against collar rot disease. Australian Journal of Crop Science, 9:69-74

Rasool SN, Jaheerunnisa S, Jayaveera KN \& Suresh Kumar C (2011) In vitro callus induction and in vivo antioxidant activity of Passiflora foetida L. leaves. International Journal of Applied Research in Natural Products, 4:01-10.
$\mathrm{R}$ development Core Team (2010) R: a language and environment for statistical computing. Vienna, R Foundation for Statistical Computing. Disponível em: <http://r-project.org>. Acessado em: 10 de setembro de 2013.

Silva AR, Rêgo ER \& Cecon PR (2011) Tamanho de amostra para caracterização morfológica de frutos de pimenteira. Horticultura Brasileira, 29:125-129.

Soares WS, Rêgo MM, Rêgo ER, Barroso PA \& Medeiros LRN (2011) Caracterização de frutos e sementes em acessos de maracujá silvestre (Passiflora foetida L.). Revista Brasileira de Plantas Medicinais, 13:569-573.

Storck L, Garcia DC, Lopes SJ \& Estefanel V (2011) Experimentação vegetal. $3^{a}$ ed. Santa Maria, UFSM. 198p.

Toebe M, Both V, Cargnelutti Filho A, Brackmann A \& Storck L (2011) Dimensionamento amostral para avaliar firmeza de polpa e cor da epiderme em pêssego e maçã. Revista Ciência Agronômica, 42:1026-1035.

Toebe M, Both V, Brackmann A, Cargnelutti Filho A \& Thewes FR (2012) Tamanho de amostra para a estimação da média de caracteres de pêssego na colheita e após o armazenamento refrigerado. Ciência Rural, 42:209-212.

Zar JH (2010) Biostatistical analysis. 5ª Ed. Upper Saddle River, Prentice Hall. 944p. 